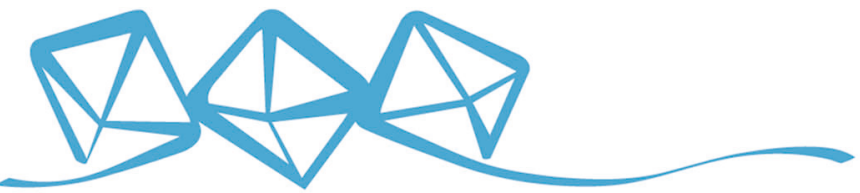 COMMUNICATIONS MATERIALS
}

ARTICLE

Check for updates

https://doi.org/10.1038/s43246-020-00090-2 OPEN

\section{Time limited self-organised criticality in the high rate deformation of face centred cubic metals}

Lewis Lea (1) ${ }^{1 凶}$, Lawrence Brown (1D ${ }^{1}$ \& Andrew Jardine ${ }^{1}$

Plastic deformation is a fundamentally important physical process, ultimately determining how materials can be used. Metal plasticity is governed by dislocation dynamics and lattice twinning. Although many continuum constitutive models exist, plasticity is now known to occur in discrete events arising from the self-organisation of dislocations into 'avalanches' under applied stress. Here we extend avalanche plasticity to high strain rates, by introducing time limitation to self-organisation. At high rates large avalanches fail to form; the system must self-organise around new constraints. Various macroscopic consequences include an increasing rate of work hardening with strain rate. We perform new measurements on high purity copper that distinguish between instantaneous and permanent strength contributions across a strength transition at $10^{4} \mathrm{~s}^{-1}$, showing the transition to be a change in structural evolution. Strong model agreement validates our time limited self-organisation approach. Our work results in a unified, physically realistic framework for plasticity, with wide applicability.

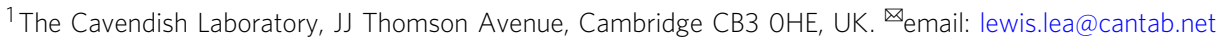


A $t$ low rates, the strength of face centred cubic (FCC) metals is renowned for being very weakly dependent on both strain rate and temperature ${ }^{1}$. Typically, strength only increases by about $10 \%$ when the deformation rate increases by an order of magnitude. However, at strain rates of about $10^{4} \mathrm{~s}^{-1}$ there is an abrupt change $\mathrm{e}^{2,3}$, above which the material strength rises around 100 times more quickly. Despite originally being observed in $1984^{2}$, the physical nature of the transition remains controversial $^{3-5}$. Studies of the effect have been limited by a lack of experimental data above the transition which can separate instantaneous strength contributions ${ }^{2,5-7}$ (e.g., due to viscous effects or phonon drag) from structural strength contributions $^{1,5,8}$ (e.g., due to changes in dislocation structure). In this paper we experimentally demonstrate the transition to be of a structural nature.

Although commonly approximated by continuum motion, at low deformation rates plastic deformation of metals is known to occur in discrete 'bursts'1,9,10. The distributions of strain burst properties $^{10,11}$, coupled with microstructural observations ${ }^{10,12}$, have led to the understanding that the underlying dislocation dynamics adopt a state of self-organised criticality ${ }^{13,14}$ (SOC), in which intermittent flow occurs by the collective slip of dislocations in 'avalanches', considered to be any slip event ranging from a single dislocation to a $3 \mathrm{D}$ collective event.

Currently, avalanche dynamics are rarely mentioned in high rate plasticity theories, and then usually only to describe the HallPetch effect ${ }^{15}$. Dislocation avalanches and SOC have been shown to influence many material properties ${ }^{1,16-18}$, most notably work hardening ${ }^{1,17,19}$. It is the indifference of SOC to external tuning which causes the initial rate of work hardening to be so weakly rate and temperature dependent ${ }^{1,11,16}$. Dislocation avalanches are also believed to contribute to macroscopic failure mechanisms such as adiabatic shear banding 20 .

SOC is observed in slowly driven, interaction dominated, threshold systems ${ }^{21}$ (SDIDTs). In order to maintain SOC the timescale of deformation must be significantly longer than both the time between two avalanches and the duration of any individual avalanche $e^{1,16,21}$. High strain-rates are therefore likely to disrupt the SOC dynamics of dislocations and have a profound effect on material behaviour. Using acoustic emission measurements from avalanche events ${ }^{10}$, Brown ${ }^{16}$ has suggested that dislocation avalanches and the consequent state of SOC will begin being disrupted at strain rates above about $10^{4} \mathrm{~s}^{-1}$. Thus, the process of time limiting SOC is a strong candidate for causing a work hardening driven strength transition around $10^{4} \mathrm{~s}^{-1}$. In contrast, the current Orowan-Taylor picture of independently slipping dislocations, adopted in most high rate plasticity models, does not predict any change in response at these rates. It is accepted that the dynamics of individual dislocations (i.e., pairwise events or 'foresting') occur on timescales ${ }^{22}$ of order $100 \mathrm{~ns}$, far shorter than that of the transition. In SOC models, all slip processes are implicitly accounted for by considering the systemwide self-organised state ${ }^{16}$. In $2 \mathrm{~d}$ simulations, Song et al. have observed that slip begins to abandon SOC above $10^{3} \mathrm{~s}^{-1}$ in favour of more 'classic' phenomenology, leading to increases in the metal's mechanical threshold ${ }^{23}$.

In order to test plasticity models at high rates, we have recently developed empirical methods ${ }^{24-26}$ that can distinguish instantaneous and structural strength contributions in uniaxial compression experiments, from the quasi-static regime, up to rates as high as $10^{5} \mathrm{~s}^{-1}$. Below $10^{4} \mathrm{~s}^{-1}$ established split Hopkinson pressure bar (SHPB) and universal testing machine methods can be used ${ }^{8,27}$. Above $10^{4} \mathrm{~s}^{-1}$, deformation must be imparted using the direct impact Hopkinson pressure bar arrangement shown in Fig. 1. The direct impact geometry allows for a rapid rise to the target strain rate. In more commonly adopted SHPB systems, dispersion during transit through the input bar shallows the rising edge of the wave, delaying specimen equilibrium at the target rate, making it impossible to obtain the very high-rate but low strain measurements that we present here. The establishment of specimen equilibrium at the target strain rate can be empirically verified through measurements of the elastic waves in both the projectile and output bars ${ }^{24,25}$, performed using photon Doppler velocimetry (PDV). Deformation can be interrupted at a particular value by introducing a tungsten-carbide strain limiting ring around the sample. To separate out instantaneous and structural strength contributions, every specimen was immediately reloaded to yield, at a much lower rate. Under the quasistatic reload conditions, any potential instantaneous strengthening mechanisms introduced in high rate measurements are removed.

Here we present measurements that show the transition to be dominated by changes in mechanical threshold, and to have temperature dependence inconsistent with mechanisms driven by phonon viscosity. A physical model is presented linking the changes in mechanical threshold to the inability of large avalanches to for rapidly enough ${ }^{16,23}$ to contribute to flow. A physical model is presented linking the changes in mechanical threshold to the inability of large avalanches to form rapidly enough to contribute to flow.

\section{Results \& Discussion}

A set of cylindrical annealed oxygen-free (grade C103) copper specimens of 16-20 $\mu \mathrm{m}$ grain size were deformed at a variety of strain rates between $10^{-2} \mathrm{~s}^{-1}$ and $10^{5} \mathrm{~s}^{-1}$. The smallest specimens were $500 \mu \mathrm{m}$ thick with a radius of $1.5 \mathrm{~mm}$. A fixed aspect ratio was used in all specimens to reduce friction effects to a constant prefactor $^{28}$. Strain was limited to $10.00 \pm 0.05 \%$, selected to minimise dynamic recovery effects ${ }^{1,8,29}$. For specimens deformed below $5 \times 10^{4} \mathrm{~s}^{-1}$, over $90 \%$ of the deformation occurred in dynamic equilibrium (i.e., after transients have settled). Reloading was performed at a strain rate of $10^{-2} \mathrm{~s}^{-1}$. Figure 2 shows the strength of each specimen immediately before (solid red circles) and after interrupt (open green circles), complete curves for a subset of experiments are shown in Supplementary Fig. 1. The upturn in strength observed above $10^{4} \mathrm{~s}^{-1}$ is retained upon reloading, and the difference between the two datasets remains consistent across the transition. The measurements indicate that the strength increase above $10^{4} \mathrm{~s}^{-1}$ is driven by a permanent structural change, evident as a strain rate dependent increase in early stage work hardening. Given these results, the relationship between SOC avalanche dynamics and strain rate can be examined around the strength transition. Strain limitation reduced adiabatic heating during high rate loading to approximately 10 $\mathrm{K}^{7,28}$. Importantly, repeat experiments at raised temperatures (Supplementary Fig. 2) show the mechanical threshold, at a fixed post-transition rate, decreases with increasing temperature, thus excluding phonon drag as a potential origin of increased work hardening in the studied regime ${ }^{7,30}$. At higher rates $\left(>10^{6} \mathrm{~s}^{-1}\right)$ or temperatures close to melt ${ }^{30-32}$, drag will likely become a dominant factor, further disrupting any remaining $\mathrm{SOC}^{23,33}$.

Experimental studies ${ }^{10,16}$ have shown that the longest timescale involved in avalanche dynamics is the time between two events, corresponding to an avalanche formation time, which is longer for larger avalanches. In strained metals, the large quantity of dislocations present will ensure the presence of a population of fully formed avalanche 'precursors', defined as any dislocation structure on the point of slip. Despite being on the point of slipping. Not all precursors are successful, other slip events in the system may arrest them, or they may slip in a non-coordinated manner, causing the dislocations involved to arrest each other 


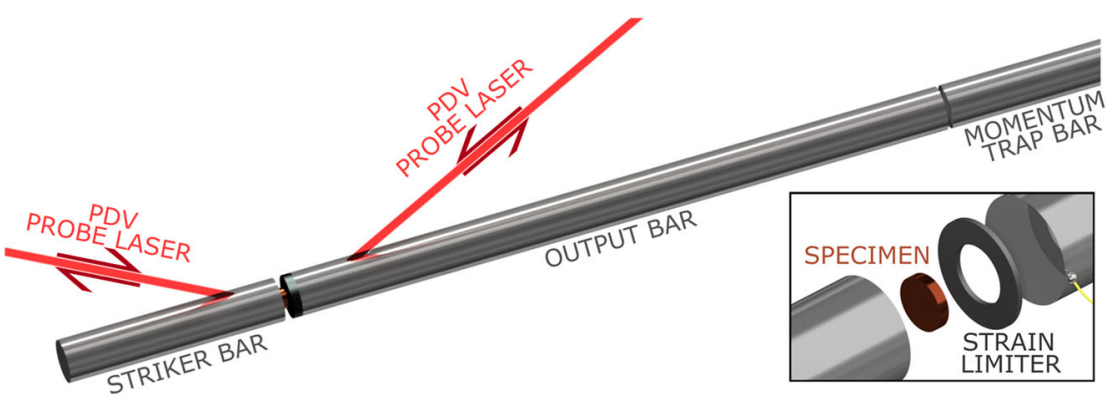

Fig. 1 A PDV instrumented direct impact Hopkinson pressure bar. Doppler interferometers measure surface velocities induced by the passage of elastic waves throughout the striker and output bars. Different linear combinations of the elastic wave measurements, combined with initial measurements of the specimen, allow the stress-strain behaviour of a material to be characterised at strain rates as high as $10^{5} \mathrm{~s}^{-1}$ without inducing shock deformation of the specimen. A tungsten carbide disk (shown in the magnified inset), mounted on the output bar interrupts deformation at $10 \%$ strain. Also shown is a yellow wire corresponding to the mounting point of thermocouples used to determine the testing temperature for experiments at nonambient temperatures.
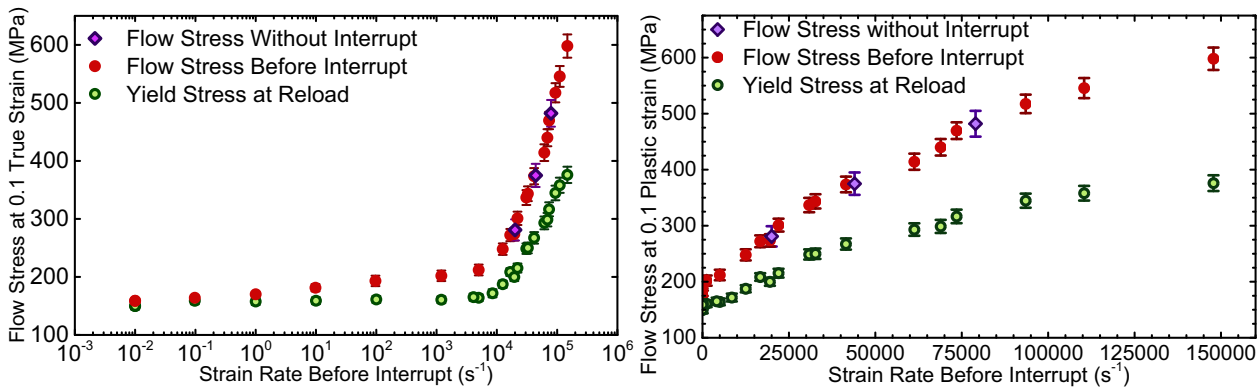

Fig. 2 The flow strength of a high purity copper during high rate deformation and at yield upon quasi-static reloading. The specimens are initially deformed at the strain rate shown on the $x$-axis to a strain of 0.1 . The solid red circles show strength measurements at the initial loading strain rate and 0.1 strain. The two-tone green measurements show the strength after reloading the interrupted specimens to yield at a fixed strain rate of $10^{-2} \mathrm{~s}^{-1}$ making the data set, to within some scaling factor, a measurement of the materials' mechanical threshold ${ }^{8}$. A small number of measurements, shown by the twotone diamond symbols, were performed without interrupt, showing the interrupting process did not affect the measurement of strength before interrupt. The transition in strength observed above $10^{4} \mathrm{~s}^{-1}$ is still present in the specimens reloaded at $10^{-2} \mathrm{~s}^{-1}$, despite the removal of variations in instantaneous effects such as phonon drag. Panel b shows the same measurements on a linear strain rate axis. Error bars for reload measurements depict fractional errors matching the deviation in interruption strain from the target strain of 0.1. The error bars in high rate measurements display the greater of the effects from either dispersive oscillations or inertial effects. Discussion of inertial effects is presented in Supplementary Methods Section 1.

immediately after slip begins. In order to perform a successful, coordinated, avalanche, all of the dislocations in a precursor must undergo some form of synchronisation. Above some critical strain rate, insufficient time will be available for precursors above a corresponding size to synchronise.

Avalanche properties, including their formation time, are observed to vary over many orders of magnitude ${ }^{10,34}$. Consequently, rather than all avalanche behaviour ceasing at single critical rate, the disruption is likely to occur progressively, with the largest avalanches failing at the lowest rates. The limit on the largest permitted avalanche corresponds to the emergence of a new length scale in the system, similar to those observed in other SOC systems when subject to a high driving rate, notably superconductor flux ${ }^{35,36}$ and water droplet ${ }^{37}$ avalanches.

The removal of the largest avalanches will cause the system to form progressively finer dislocation networks at equivalent strain, increasing the rate of work hardening. Such a mechanism is consistent with microstructural observations ${ }^{38}$. Close to the transition rate, specimens deformed to fixed strain at increasing strain rates show a progressive replacement of organised cellular structures with random distributions of dislocations ${ }^{39}$; a direct consequence of disrupting collective motion. Furthermore, at higher rates increased numbers of lone dislocations are found enclosed within dislocation cells, likely those left out of the collective activation.
Avalanche interactions with different impurities and microstructures will lead to transitions of varying magnitude and onset rate. In the slowly driven regime, the size of the largest occurring avalanche (and consequently the transition rate) will be determined by some spatial limitation. Spatially limiting avalanches is expected to strengthen the material as observed in multiple scenarios; the Hall-Petch effect ${ }^{15}$, nanopillar deformation ${ }^{40}$ and dispersion hardening ${ }^{41}$. The latter two cases exhibit clear increases in work hardening. If the spatial limit is lowered (e.g., by reducing the grain size), the transition to the time limited avalanche regime will shift to higher rates, potentially vanishing at the lowest grain sizes. Casem et al. $^{42}$ have observed such a phenomenon in aluminium and Mao et al. $^{43}$ in copper, the same is observed in alloys ${ }^{44,45}$. The reverse also holds; as long as the time limited regime is maintained, changes in spatial limitation should have no effect. Park et al. ${ }^{46}$ have observed that at true strain rates of $10^{7} \mathrm{~s}^{-1}$, grain size effects vanish above $250 \mathrm{~nm}$, the smallest they measured.

More detailed study of avalanches themselves is required to describe the relation between avalanche dynamics and the consequent rate of work hardening. As an illustrative example of the significance of $3 \mathrm{D}$ avalanches in SOC, we will discuss the Brown model of avalanche plasticity ${ }^{1,16}$, where avalanches correspond to slip bands caused by the rapid planar expansion of 'blade like' (1:30 aspect ratio) Eshelby inclusions ${ }^{47}$, bound by a surface of 
both edge and screw dislocations. The ellipsoidal inclusion geometry ensures that all of the surface dislocations experience the same stress ${ }^{47,48}$, a requirement for collective slip. If the ellipsoidal slip band is confined to one plane, it becomes identical to the dislocation pile up of Eshelby, Frank and Nabarro ${ }^{49}$.

Dislocations in 3D slip bands and their precursors experience greatly magnified forces compared to an individual dislocation ${ }^{1,50}$. In pure FCC metals the resultant force is sufficient to sweep away many of the forest dislocations they meet. Successive avalanches turn the lattice into a palimpsest of dislocation structures; mostly overwriting each other but allowing a small fraction of earlier structures to remain. The presence of screw dislocations at the bounding surface, combined with the extension of the inclusion across many crystal planes, allows the band to pivot very slightly about its axes. The departure from the lattice plane forces the activation of new secondary slip throughout the band as it propagates, anchoring the band after it reaches a sufficient size. Using such a collective mechanism, and the powerful symmetry laws present in SOC, a complete set of 3D avalanche properties and consequently physically realistic material behaviours can be derived, matching both quantitative and microstructural observations ${ }^{1,16}$.

Of current interest is the initial rate of work hardening, $\Theta$, derived by Brown ${ }^{1}$ to follow the relation

$$
\Theta=\frac{\delta \sigma_{M T S}}{\delta \varepsilon_{p}} \propto \frac{1}{\psi} .
$$

$\psi$ is the volume fraction of the lattice eligible for slip via any mechanism, strongly linked to both stress and strain fluctuations through scale invariance in the system due to SOC ${ }^{1}$. The strength metric we adopt in the current discussion is the mechanical threshold stress ${ }^{8}, \sigma_{\mathrm{MTS}}$; defined as the flow stress the material would have if it were deformed at $0 \mathrm{~K}$, removing ambiguities arising from the variation of temperature and strain rate during any particular part of an experiment. In the slowly driven case ${ }^{1}$, the slip eligible volume fraction is the same as the obstacle-free volume fraction. In the time-limited regime, unviable precursors no longer contribute to the slip eligible volume, so the eligible volume fraction progressively decreases with an increasing rate. The general slip eligible volume is computed as a summation over the volumes of all dislocation structures on the point of slip.

The scale-free nature of the system constrains all axes of any avalanche precursor to be proportional to one another. The precursor can therefore be described by a single generic length scale, L. The probability distribution of avalanche lengths has been empirically ${ }^{10}$ and theoretically ${ }^{16}$ determined to follow the relation $P(L) \propto L^{-2} \mathrm{~d} L$. Consequently, whilst the largest avalanches only account for a small fraction of slip events, they provide a much larger contribution to the slip eligible volume, $L^{3} P(L) \propto L \mathrm{~d} L$, and thus the self-organisation of the microstructure. Computing the slip eligible volume fractions in the spatially limited (signified by a subscript 0 ) and time-limited scenarios, a power-law relation can be found (see Supplementary Methods Section 2 for details) between work hardening and the generic length of the largest viable avalanche, $\mathrm{L}_{\mathrm{L}}$;

$$
\Theta=\Theta_{0} \frac{\psi_{0}}{\psi} \approx \Theta_{0}\left(\frac{L_{L_{0}}}{L_{L}}\right)^{2} .
$$

The measured probability distribution of strain fluctuations has shown strain increments imparted by avalanches to be proportional to the generic length scale squared ${ }^{1}\left(\Delta y \propto L_{0}^{2}\right)$. An equivalent statement to the derived relation is therefore that the rate of work hardening is approximately inversely proportional to the largest possible slip increment, as observed by Csikor et al. ${ }^{17}$.
To relate work hardening to strain rate we must now estimate the synchronisation time for an avalanche. A simplified schematic of an avalanche event is shown in Fig. 3. The participating dislocations are analogous to a drum skin, held taught under stress, and plucked by the sudden overcoming of an obstacle. We wish to find the time required to settle to a motion which will allow every dislocation to expand at the same time. Due to the atomic structure of the dislocations, we wish to find the time to synchronise the atomic sites on the ellipsoidal surface, proportional to $L^{2}$. Communication between different dislocations requires transverse oscillations. The transverse oscillation period of a dislocation is proportional to its line length, thus proportional to $L$, divided by the shear wave speed of the medium, $C_{s}$. Simple arguments, therefore, provide a scaling relation between an avalanche size and its synchronisation time; $t_{f} \propto L^{3} C_{\mathrm{s}}{ }^{-1}$. For our ellipsoidal example, more detailed consideration (Supplementary Methods Section 3), produces the approximation

$$
t_{f} \approx \sqrt{2 \pi} \frac{4 \pi}{3} \frac{L_{c} L_{a}^{2}}{d b C_{S}},
$$

where $b$ is the length of the Burger's vector and $d$ is the interplanar spacing of the relevant slip system. $L_{a}$ is the major axis of the ellipsoid, corresponding to the axes which lie in the avalanche slip plane, $L_{c}$ is the minor, or slip plane normal axis $\left(L_{\mathrm{a}} \approx 30 L_{\mathrm{c}}\right)$.

The slip-normal axis remains unchanged after the avalanche, and thus can be inferred from the measurements of the resultant slip bands ${ }^{29,51}$ to be in the range $3.1-10 \mathrm{~nm}$. The transient nature of the precursor axes in the slip plane prevents them from being measured.

However, the length of the largest in-plane axes can be inferred to be in the range $100-300 \mathrm{~nm}$ due to the $30: 1$ aspect ratio of the precursor, determined using the deviation of resulting slip bands from crystallographic slip planes ${ }^{1}$. Combined with standard lattice and shear wave measurements for copper, the synchronisation time for the largest avalanche is predicted to lie in the range 2 to $80 \mu \mathrm{s}$, depending on the exact dimensions of its axes. The predicted synchronisation times are consistent with the observed time between avalanches in acoustic emission ${ }^{10}$ and the delay between stress collapse and temperature rise in adiabatic shear banding ${ }^{52}$. Whilst the geometric prefactors presented are specific to the Eshelby inclusion, the $L^{3} C_{\mathrm{s}}{ }^{-1}$ scaling is general to all $3 \mathrm{D}$ precursors, as is the spatial limitation to $L$, consequently all $3 \mathrm{D}$ mechanisms will have similar synchronisation times and in SOC, the same scaling law.

3D scaling laws help understand observations by Armstrong ${ }^{15}$, that the transition could correspond to an asymptotic reduction in activation volume for slip by a factor of order 1000. Avalanche behaviour increases both the number of dislocations undergoing slip, and, by the same factor, the distance the band travels before arresting itself. Recalling the, largest slip bandwidth is typically 30 planes, and thus involves 30 dislocations. The activation volume parameter is therefore expected to be 900 times that of a single dislocation. Progressive avalanche breakdown to the point where only individual slip remains possible provides a quantitatively accurate mechanism to explain Armstrong's observations. Furthermore, activation volumes display the same time and spatial limit interplay as many other material properties. A rapid drop in activation volume is observed for decreasing grain sizes in copper $^{53}$ beginning at grain sizes of approximately $300 \mathrm{~nm}$; close to the largest observed in-plane avalanche axes.

The time available for synchronisation is inversely proportional to the strain rate. Combining the relations linking work hardening and synchronisation time to the generic avalanche length 


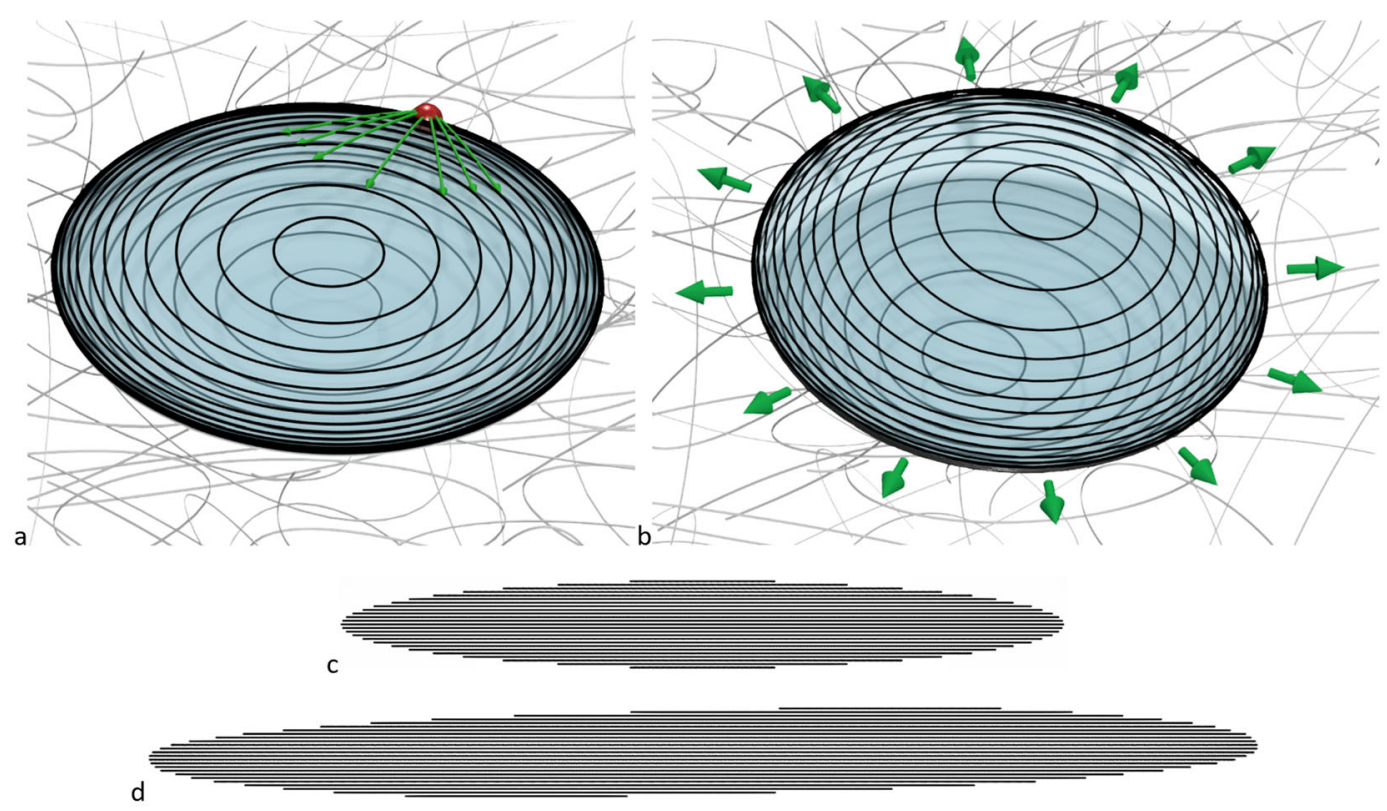

Fig. 3 The stages of an Eshelby inclusion avalanche. After a number of small slip events, an arbitrary forest of individually pinned dislocations form a population of volumetric Eshelby inclusions (a) bound by a surface of dislocations. The volumetric slip of the inclusion is capable of imparting much greater strain and activating at much lower applied stress than an individual dislocation, as all dislocations involved contribute to overcoming the obstacle; the red sphere in (a). In order to slip collectively, the dislocations binding the inclusion must synchronise their motion against the obstacle, requiring the propagation of information between the organising dislocations (a). The scaling of the surface and the transverse oscillation period of the binding dislocations will cause the synchronisation time to scale in approximate proportion to the inclusion volume. Avalanches that manage to synchronise collectively slip as shown in (b), both expanding and tilting the inclusion, imparting a large volumetric strain into the lattice that scales with inclusion size. The side profiles of the inclusion are shown (not to scale) before slip in (c) and after in (d). The volumetric shear, combined with the mobility of screw dislocations in the interface, enables the avalanche axes to depart from the crystal plane. The projection of applied stresses on to secondary systems eventually activates them, anchoring the band?

scale, we arrive at the expression

$$
\begin{aligned}
\Theta(\dot{\varepsilon}, T) & \approx \Theta_{0} \times \operatorname{Max}\left[1,\left(\frac{C_{s_{0}}(T) t_{0}(T)}{C_{s}(T) t}\right)^{2 / 3}\right] \\
& \approx \Theta_{0} \times \operatorname{Max}\left[1,\left(\lambda \frac{\dot{\varepsilon}}{C_{s}(T)}\right)^{2 / 3}\right] .
\end{aligned}
$$

The max function captures the transition from spatial to timelimited organisation, values with a subscript 0 again correspond to those at the transition. $\dot{\varepsilon}_{0}(T)$ is the predicted transition rate as a function of temperature. For a single material, the ratio $\dot{\varepsilon}_{0}(T) C_{S}(T)^{-1}$ is expected to be constant and thus is replaced by $\lambda$. The approximate equality arises due to the neglect of the minimum possible size of a slip band (a single dislocation). In the more precise form (equation 8 in the supplementary methods), when the minimum permitted avalanche size passes through the size of a single dislocation, physically corresponding to none of the metal being slip eligible, the rate of work hardening diverges. In a real material the main mode of deformation will change to other mechanisms such as twinning, which is expected at higher strains, higher strain rates, or lower temperatures for the current grain size 7,54 , or nucleation limited processes expected at substantially higher stresses ${ }^{55}$. However, the current theory could be used to predict the point at which such changes should occur.

An important feature of the current model is that at no point have any observations or measurements from above the transition been used, the proposed transition rates and scaling laws have been derived purely using the properties of scale invariance, microstructural observations from quasi-statically deformed specimens and acoustic emission measurements, also performed in quasi-static deformation.

The form presented in equation 5 is not directly comparable to the measurements presented in Fig. 2; the flow stress of specimens was measured, not their initial rate of work hardening. Whilst efforts were made to minimise the effects of dynamic recovery during work hardening they must still be accounted for. In order to separate these effects, the measurements presented in Fig. 2 were repeated with interruption at true strains between 0 and 1 , and temperatures between ambient and $300^{\circ} \mathrm{C}$. These measurements and their subsequent use to extract $\Theta(\dot{\varepsilon}, T)$ are presented in the section 4 of the supplementary methods. An important observation from the expanded measurement set is that no significant change in behaviour ${ }^{56}$ occurs in the saturation stress of the metal above $10^{4} \mathrm{~s}^{-1}$. If one were to attempt to model the increase in work hardening as a simple increase in the generation of dislocations during deformation, then the plateau stress, often corresponding to creation-annihilation equilibrium in said pictures, would also be expected to change. The fact that the increase in work hardening is not accompanied by a change in saturation stress signifies a separation between the processes of work hardening and dynamic recovery. Our current understanding would suggest they are separated by scale; work hardening occurs at the system scale (SOC), whilst saturative mechanisms (crossslip, annihilation, exhaustion) occur close to the atomic scale. Furthermore, this separation explains the common observation that the rate dependent strengthening observed above $10^{4} \mathrm{~s}^{-1}$ decreases with increasing strain or mechanical threshold ${ }^{15,57-63}$, emphasising the importance of studying annealed or low-worked specimens ${ }^{15,60-63}$. 

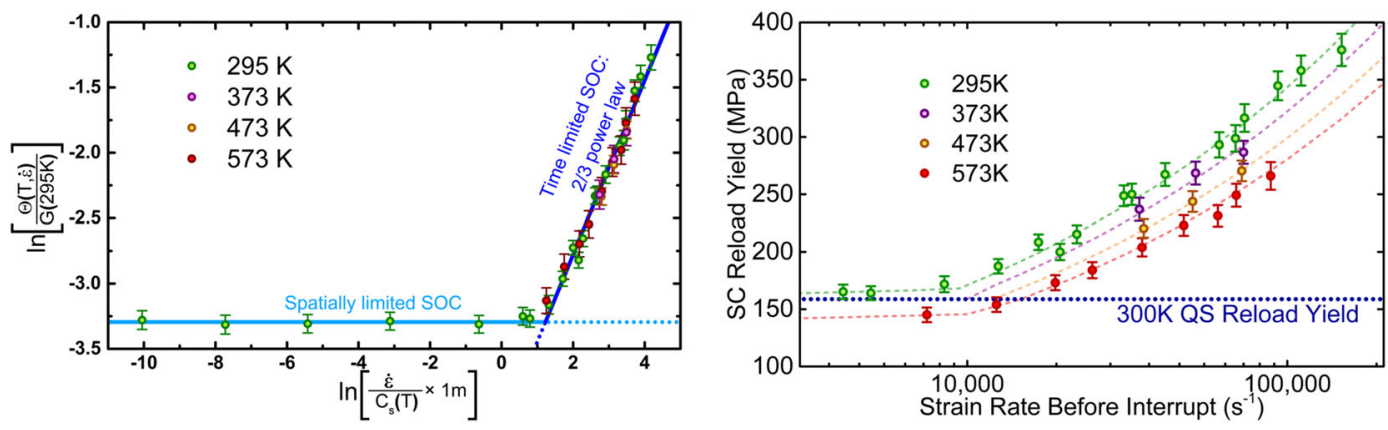

Fig. 4 A work hardening master curve above the strength tranistion. The initial rate of work hardening in the mechanical threshold stress measured for a variety of rates between $10^{-2} \mathrm{~s}^{-1}$ and $10^{5} \mathrm{~s}^{-1}$, at four temperatures. A small correction is made to strain rate to account for variations in sound speed with temperature. The measurements collapse on to a single curve, which displays a 2/3 power law, predicted by modelling the breakdown of 3D dislocation avalanches in the system. The rate of work hardening is obtained from reload measurements performed at a variety of strain rates, strains and temperatures, using analysis outlined in the section 4 of the supplementary methods. Panel b shows the reload measurements for 0.1 true strain at each rate and temperature studied, dashed lines signify the reload stresses predicted by the model. The extracted model parameters are $\Theta_{0}=1.68 \pm 0.03 \mathrm{GPa}$ and $\lambda=3.03 \pm 0.07 \mathrm{~m}$. At $295 \mathrm{~K}$ the transition occurs at $6900 \pm 300 \mathrm{~s}^{-1}$. Error bars for extracted measurements of $\Theta$ are standard errors from the fitting process outlined in Supplementary Methods Section 4. Error bars in the reload measurements correspond to fractional errors matching the deviation in the measured interruptions strain from the 0.1 target interrupt strain.

Figure 4 shows the measured rates of work hardening, normalised to the shear modulus during reloading, as a function of strain rate, normalised to the sound speed during the initial loading, for a number of temperatures. After accounting for saturation and sound speed variations, the measurements collapse into a master curve. Presented in logarithmic space, the posttransition regime shows a good linear fit with gradient $0.68 \pm$ 0.04 , agreeing with the proposed 2/3 power law to within its error. Plotting the normalised strain rate to the power of $2 / 3$ against $\Theta$ normalised to $G(T)$ provides a linear relation which if extrapolated to zero rate passes through the origin to within its own error. Both features show strong agreement with the model.

The solid lines show the complete model (equation 5) across both regimes, and accurately depict the entire data set using the single fitting parameter $\lambda$ which was inferred from the room temperature data set alone. Whilst the exact value of $\lambda$ cannot yet be predicted, its physical origin is well established and its order of magnitude can be predicted from acoustic emission experiments ${ }^{16}$. Future development of the model could aim to predict this parameter as well, by considering the contributions of grain size, specimen size, impurity population and type to the confinement of avalanches. The current model accurately reproduces reload stresses for all available temperatures, rates and strains, using only room temperature data and an accurate model for the saturation stress.

Overall, the proposed model unifies low-rate avalanche plasticity and dynamic plasticity. Based on the synchronisation of dislocations into avalanches and their subsequent self-organisation, the model provides accurate qualitative and quantitative predictions of the behaviour of high purity FCC metals at high strain rates. The transition rate can be approximated and correct scaling laws of the material behaviour can be obtained using information gained purely from low rate deformation. Improved understanding of the synchronisation and activation of dislocation avalanches should aid the metallurgical design of high impact resistant structures, micro-scale forming ${ }^{17}$ and ultra-fine grained metals ${ }^{46}$. Studying the breakdown of self-organisation in dislocation systems as they cease being slowly driven can improve our understanding of the nature of SOC itself, and thus our ability to predict the behaviour of complex, and often experimentally inaccessible ${ }^{21}$ systems throughout all fields of scientific study.

\section{Data availability}

The datasets presented in this article can be found at https://doi.org/10.17863/ CAM. 57308 .

Received: 2 September 2019; Accepted: 14 October 2020; Published online: 01 December 2020

\section{References}

1. Brown, L. M. Constant intermittent flow of dislocations: central problems in plasticity. Mater. Sci. Technol. 28, 1209-1232 (2012).

2. Follansbee, P., Regazzoni, G. \& Kocks, U. The transition to drag-controlled deformation in copper at high strain rates. Mech. Prop. High Rates Strain 70, $71-80$ (1984)

3. Jordan, J. L., Siviour, C. R., Sunny, G., Bramlette, C. \& Spowart, J. E. Strain rate-dependant mechanical properties of OFHC copper. J. Mater. Sci. 48, 7134-7141 (2013).

4. Salvado, F. C., Texeira-Dias, F., Walley, S. M. \& Lea, L. J. \& CardosoJ.B. A review on the strain rate dependency of the dynamic viscoplastic response of FCC metals. Prog. Mater. Sci. 88, 186-231 (2017).

5. Lea, L. J. \& Walley, S. M. High Strain Rate Metal Plasticity. in Encyclopedia of Continuum Mechanics 1-20 (Springer Berlin Heidelberg, 2019). https://doi. org/10.1007/978-3-662-53605-6_219-1.

6. Hunter, A. \& Preston, D. L. Analytic model of the remobilization of pinned glide dislocations from quasi-static to high strain rates. Int. J. Plast. 70, 1-29 (2015).

7. Lea, L. J. \& Jardine, A. P. Characterisation of high rate plasticity in the uniaxial deformation of high purity copper at elevated temperatures. Int. J. Plast. 102, 41-52 (2018).

8. Follansbee, P. S. \& Kocks, U. F. A constitutive description of the deformation of copper-based on the use of the mechanical threshold stress as an internal state variable. Acta Metall 36, 81-93 (1988).

9. Tinder, R. F. \& Trzil, J. P. Millimicroplastic burst phenomena in zinc monocrystals. Acta Metall 21, 975-989 (1973).

10. Weiss, J. et al. Evidence for universal intermittent crystal plasticity from acoustic emission and high-resolution extensometry experiments. Phys. Rev. B 76, 224110 (2007)

11. Dimiduk, D. M., Woodward, C., LeSar, R. \& Uchic, M. D. Scale-free intermittent flow in crystal plasticity. Science 312, 1188-1190 (2006)

12. Sprušil, B. \& Hnilica, F. Fractal character of slip lines of Cd single crystals. Czechoslov. J. Phys. B 35, 897-900 (1985).

13. Bak, P., Tang, C. \& Wiesenfeld, K. Self-organized criticality: an explanation of the 1/f noise. Phys. Rev. Lett. 59, 381-384 (1987).

14. Bak, P. \& Weissman, M. How nature works: the science of self-organized criticality. Am. J. Phys 65, 579-580 (1997).

15. Armstrong, R. \& Walley, S. High strain rate properties of metals and alloys. Int. Mater. Rev. 82, 073908 (2008). 
16. Brown, L. M. Power laws in dislocation plasticity. Philos. Mag. 96, 2696-2713 (2016).

17. Csikor, F. F., Motz, C., Weygand, D., Zaiser, M. \& Zapperi, S. Dislocation avalanches, strain bursts, and the problem of plastic forming at the micrometer scale. Science 318, 251-254 (2007).

18. Dimiduk, D. M., Uchic, M. D., Rao, S. I., Woodward, C. \& Parthasarathy, T. A. Overview of experiments on microcrystal plasticity in fcc-derivative materials: Selected challenges for modelling and simulation of plasticity. Model. Simul. Mater. Sci. Engng 15, 135-146 (2007).

19. Brown, L. M. Chapter 58 Linear work-hardening and secondary slip in crystals. Dislocations in Solids 11, 193-210 (2002).

20. Armstrong, R. W., Coffey, C. S. \& Elban, W. L. Adiabatic heating at a dislocation pile-up avalanche. Acta Met 30, 2111-2116 (1982).

21. Jensen, H. J. Self-organized criticality: emergent complex behavior in physical and biological systems. (Cambridge University Press, 1998).

22. Gray, G. T. Material response to shock/dynamic loading: windows into kinetic and stress-state effects on defect generation and damage evolution. in. AIP Conference Proceedings 1426, 19-26 (2012).

23. Song, H., Dimiduk, D. \& Papanikolaou, S. Universality class of nanocrystal plasticity: localization and self-organization in discrete dislocation dynamics. Phys. Rev. Lett. 122, 178001 (2019).

24. Lea, L. \& Jardine, A. Measuring structural evolution in the dynamic plasticity of FCC metals. AIP Conf. Proc. 1979, 70020 (2018).

25. Lea, L. J. \& Jardine, A. P. Application of photon Doppler velocimetry to direct impact Hopkinson pressure bars. Rev. Sci. Instrum. 87, 023101 (2016).

26. Morley, O. J. \& Williamson, D. M. Pressure and temperature induced red-shift of the sodium D-line during HMX deflagration. Commun. Chem. 3 (2020).

27. ASM Handbook Vol 8: Mechanical Testing and Evaluation. in Hardness testing, ASM International (eds Kuhn, H. \& Medlin, D.) (2000).

28. Gorham, D., Pope, P. \& Field, J. An improved method for compressive stressstrain measurements at very high strain rates. Proc. R. Soc. A Math. Phys. Eng. Sci. 438, 153-170 (1992).

29. Kocks, U. F. \& Mecking, H. Physics and phenomenology of strain hardening: the FCC case. Prog. Mater. Sci. 48, 171-273 (2003).

30. Frutschy, K. J. \& Clifton, R. J. High-temperature pressure-shear plate impact experiments on of hc copper. J. Mech. Phys. Solids 46, 1723-1744 (1998).

31. Zaretsky, E. B. \& Kanel, G. I. Effect of temperature, strain, and strain rate on the flow stress of aluminum under shock-wave compression. J. Appl. Phys. 112, 073504 (2012).

32. Grunschel, S., Clifton, R. J. \& Jiao, T. Shearing resistance of aluminum at high strain rates and at temperatures approaching melt. AIP Conf. Proc 1426, 1335-1338 (2012).

33. Papanikolaou, S. et al. Quasi-periodic events in crystal plasticity and the selforganized avalanche oscillator. Nature 490, 517-521 (2012).

34. Weiss, J., Lahaie, F. \& Grasso, J. R. Statistical analysis of dislocation dynamics during viscoplastic deformation from acoustic emission. J. Geophys. Res. Solid Earth 105, 433-442 (2000).

35. Field, S., Witt, J., Nori, F. \& Ling, X. Superconducting vortex avalanches. Phys. Rev. Lett. 74, 1206-1209 (1995).

36. Yeh, W. J. \& Kao, Y. H. Flux-flow noise in type-II superconductors. Phys. Rev. B 44, 360-373 (1991).

37. Plourde, B., Nori, F. \& Bretz, M. Water droplet avalanches. Phys. Rev. Lett. 71, 2749-2752 (1993).

38. Gray, G. T. III High-strain-rate deformation: mechanical behavior and deformation substructures induced. Annu. Rev. Mater. Res. 42, 285-303 (2012).

39. Kiritani, M. et al. Defect structures introduced in FCC metals by high-speed deformation. Radiat. Eff. Defects Solids 157, 53-74 (2002).

40. Dimiduk, D. M., Uchic, M. D. \& Parthasarathy, T. A. Size-affected single-slip behavior of pure nickel microcrystals. Acta Mater. 53, 4065-4077 (2005).

41. Ashby, M. F. Work hardening of dispersion-hardened crystals. Philos. Mag 14, 1157-1178 (1966).

42. Casem, D. T., Ligda, J. P., Schuster, B. E. \& Mims, S. High-Rate Mechanical Response of Aluminum Using Miniature Kolsky Bar Techniques. in Dynamic Behavior of Materials, Volume 1: Proceedings of the 2017 Annual Conference on Experimental and Applied Mechanics (eds Kimberley, J., Lamberson, L. \& Mates, S.) 147-153 (Springer International Publishing, 2018). https://doi.org/ 10.1007/978-3-319-62956-8_25.

43. Mao, Z. N., An, X. H., Liao, X. Z. \& Wang, J. T. Opposite grain size dependence of strain rate sensitivity of copper at low vs high strain rates. Mater. Sci. Eng. A 738, 430-438 (2018).

44. Casem, D., Ligda, J., Walter, T., Darling, K. \& Hornbuckle, B. Strain-rate sensitivity of nanocrystalline $\mathrm{Cu}-10 \mathrm{Ta}$ to $700,000 /$ s. J. Dyn. Behav. Mater 6 24-33 (2020)

45. Turnage, S. A. et al. c. Nat. Commun. 9 (2018)
46. Park, H. S. et al. Grain-size-independent plastic flow at ultrahigh pressures and strain rates. Phys. Rev. Lett. 114, 065502 (2015).

47. Eshelby, J. D. The determination of the elastic field of an ellipsoidal inclusion, and related problems. Proc. R. Soc. London A Math. Phys. Eng. Sci. 241, 376-396 (1957)

48. Markenscoff, X. Inclusions with constant eigenstress. J. Mech. Phys. Solids $\mathbf{4 6}$ 2297-2301 (1998)

49. Eshelby, J. D., Frank, F. C. \& Nabarro, F. R. N. XLI. The equilibrium of linear arrays of dislocations. London, Edinburgh, Dublin Philos. Mag. J. Sci 42, 351-364 (1951).

50. Brown, L. M. Dislocation pile-ups, slip-bands, ellipsoids, and cracks. Mater. Sci. Eng. A 400, 2-6 (2005).

51. Neuhauser, H. Slip-line formation and collective dislocation motion. Dislocations in solids 6, 319-440 (1983).

52. Guo, Y. et al. Temperature Rise Associated with Adiabatic Shear Band: Causality Clarified. Phys. Rev. Lett. 122 (2019).

53. Wang, Y., Liu, Y. \& Wang, J. T. Investigation on activation volume and strainrate sensitivity in ultrafine-grained tantalum. Mater. Sci. Eng. A 635, 86-93 (2015).

54. Meyers, M. A., Vöhringer, O. \& Lubarda, V. A. The onset of twinning in metals: a constitutive description. Acta Mater 49, 4025-4039 (2001).

55. Armstrong, R. W., Arnold, W. \& Zerilli, F. J. Dislocation mechanics of copper and iron in high rate deformation tests. J. Appl. Phys. 105, 23511 (2009).

56. Lea, L. Structural Evolution in the Dynamic Plasticity of FCC Metals. (University of Cambridge, 2017). https://doi.org/10.17863/CAM.20971.

57. Follansbee, P. S. LA-UR-85-3026: HIGH STRAIN RATE DEFORMATION IN FCC METALS AND ALLOYS. in Explomet '85', International Conference on Metallurgical Applications of Shock-Wave and High-Strain-Rate Phenomena (1985). https://permalink.lanl.gov/object/tr?what=info:lanl-repo/lareport/LAUR-85-3026.

58. Langer, J. S. Thermodynamic theory of dislocation-enabled plasticity, https:// doi.org/10.1103/PhysRevE.96.053005 (2017).

59. Rosenberg, Z., Kositski, R., Ashuach, Y., Leus, V. \& Malka-Markovitz, A. On the upturn phenomenon in the strength vs. strain-rate relations of metals. Int. J. Solids Struct. 176-177, 185-190 (2019).

60. Brown, L. M. Unifying concepts in dislocation plasticity. Philos. Mag. 85, 2989-3001 (2005)

61. Suzuki, T., Takeuchi, S. \& Yoshinaga, H. Dislocation Dynamics and Plasticity. (Springer-Verlag Berlin Heidelberg, 1991). https://doi.org/10.1007/978-3-642 75774-7.

62. Gould, P. J. \& Goldthorpe, B. D. A path-dependent constitutive model for gilding copper. J. Phys. IV 10 (2000).

63. Forde, L. C., Proud, W. G. \& Walley, S. M. Symmetrical Taylor impact studies of copper. Proc. R. Soc. London A Math. Phys. Eng. Sci. 465, 769-790 (2009).

\section{Acknowledgements}

This work was funded by the Engineering and Physical Sciences Research Council and QinetiQ. We would like to thank P. Church and P. Gould for their ongoing interest and discussion. They would also like to thank the Cavendish workshop for preparing experimental specimens.

\section{Author contributions}

L.J.L. and L.M.B. developed the analytical model of time-limited self-organisation. L.J.L. and A.P.J. developed the P.V.D. and D.I.H.B. apparatus. L.J.L. performed experimental strength measurements. All authors contributed to the paper.

\section{Competing interests}

The authors declare no competing interests.

\section{Additional information}

Supplementary information is available for this paper at https://doi.org/10.1038/s43246 020-00090-2.

Correspondence and requests for materials should be addressed to L.L.

Peer review information Primary handling editor: John Plummer

Reprints and permission information is available at http://www.nature.com/reprints

Publisher's note Springer Nature remains neutral with regard to jurisdictional claims in published maps and institutional affiliations. 
(c) (i) Open Access This article is licensed under a Creative Commons Attribution 4.0 International License, which permits use, sharing, adaptation, distribution and reproduction in any medium or format, as long as you give appropriate credit to the original author(s) and the source, provide a link to the Creative Commons license, and indicate if changes were made. The images or other third party material in this article are included in the article's Creative Commons license, unless indicated otherwise in a credit line to the material. If material is not included in the article's Creative Commons license and your intended use is not permitted by statutory regulation or exceeds the permitted use, you will need to obtain permission directly from the copyright holder. To view a copy of this license, visit http://creativecommons.org/ licenses/by/4.0/.

(C) The Author(s) 2020 